\title{
Superstability of approximate d'Alembert harmonic functions
}

\author{
Hark-Mahn Kim¹, Gwang Hui Kim² and Mi Hyun $\operatorname{Han}^{1 *}$
}

\author{
* Correspondence: good1014@cnu. \\ ac.kr \\ 'Department of Mathematics, \\ Chungnam National University, 79 \\ Daehangno, Yuseong-gu, Daejeon \\ 305-764, Korea \\ Full list of author information is \\ available at the end of the article
}

\section{Abstract}

In this article, we study the superstability problem for the complex-valued functional equation

$$
f(x+y+z)+f(x+y-z)+f(y+z-x)+f(z+x-y)=4 f(x) f(y) f(z)
$$

on an abelian group and on a commutative semisimple Banach algebra. As a result, we obtain application to harmonic functions satisfying the equation approximately.

\section{Introduction}

The problem of stability of functional equations was originally stated by Ulam [1]. For Banach spaces, Hyers [2] gave the first partial solution to Ulam's question in 1941, which states that if $\delta>0$ and $f: X \rightarrow Y$ is a mapping, where $X, Y$ are Banach spaces, such that

$$
\|f(x+y)-f(x)-f(y)\| \leq \delta
$$

for all $x, y \in X$, then there exists a unique additive mapping $T: X \in Y$ such that

$$
\|f(x)-T(x)\| \leq \delta
$$

for all $x \in X$. And then Aoki [3] and Bourgin [4] generalized the theorem of Hyers by considering the stability problem with unbounded Cauchy differences for approximately additive mappings. Rassias [5] succeeded in extending the result of Hyers for approximate linear mappings by weakening the condition for the Cauchy difference to be unbounded. The stability phenomenon that was presented by Rassias may be called the generalized Hyers-Ulam stability. This terminology may also be applied to the cases of other functional equations (see [6]). The stability problem for functional equations has been extensively investigated by a number of mathematicians [7-15]. On the other hand, there is a strong stability phenomenon which is known as a superstability. An equation of a homomorphism is called superstable if each approximate homomorphism is actually a true homomorphism. This property was first observed when the following theorem was proved by Baker et al. [16].

Theorem 1.1. Let $V$ be a vector space. If a function $f: V \rightarrow \mathbf{R}$ satisfies the inequality

$$
|f(x+y)-f(x) f(y)| \leq \varepsilon
$$


for some $\varepsilon>0$ and for all $x, y \in V$, then either $f$ is bounded or $f(x+y)=f(x) f(y)$ for all $x, y \in V$.

In 1980, Baker [17] reported the stability of the cosine functional equation

$$
f(x+y)+f(x-y)=2 f(x) f(y)
$$

which is also called the d'Alembert equation, as in the following theorem.

Theorem 1.2. If $\delta>0, G$ is an abelian group and $f$ is a function such that

$$
|f(x+y)+f(x-y)-2 f(x) f(y)| \leq \delta
$$

for all $x, y \in G$, then either $f$ is bounded by the constant $\frac{1+\sqrt{1+2 \delta}}{2}$ or $f$ satisfies the d'Alembert functional equation (1) for all $x, y \in G$.

In 2002, Badora and Ger [18] proved the superstability of d'Alembert functional equation concerning complex-valued mappings.

Theorem 1.3. Let $(G,+)$ be an abelian group. Let $f: G \rightarrow C$ and $\varphi: G \rightarrow[0, \infty)$ satisfy one of the inequality

$$
|f(x+y)+f(x-y)-2 f(x) f(y)| \leq \phi(x) \quad \forall x, y \in G
$$

or

$$
|f(x+y)+f(x-y)-2 f(x) f(y)| \leq \phi(x) \quad \forall x, y \in G .
$$

Then, either $f$ is bounded or $f$ satisfies Equation 1 .

Theorem 1.4. Let $(G,+)$ be an Abelian group and let $(\mathcal{A},\|\cdot\|)$ be a semisimple commutative Banach algebra. Assume that $f: G \rightarrow \mathcal{A}$ and $\varphi: G \rightarrow \mathbf{R}$ satisfy anyone of the inequalities

$$
\|f(x+y)+f(x-y)-2 f(x) f(y)\| \leq \phi(\gamma)
$$

or

$$
\|f(x+y)+f(x-y)-2 f(x) f(y)\| \leq \phi(x)
$$

for all $x, y \in G$. Then,

$$
f(x+y)+f(x-y)=2 f(x) f(y)
$$

for all $x, y \in G$, provided that for an arbitrary linear multiplicative functional $x^{*} \in \mathcal{A}^{*}$ the superposition $x^{*} \circ f$ fails to be bounded.

Remark 1.5. Now, we consider the following example. Let $\mathcal{A}$ be a Banach algebra of all diagonal $2 \times 2$ matrices with complex entries and let $\bar{f}: \mathbf{R} \rightarrow \mathcal{A}$ be given by the formula

$$
\bar{f}(x):=\left[\begin{array}{cc}
f(x) & 0 \\
0 & b
\end{array}\right],
$$

where $f: \mathbf{R} \rightarrow \mathcal{A}$ is an unbounded solution to (1) and $b \in \mathbf{C} \backslash\{0,1\}$ is arbitrarily fixed. Then, $\bar{f}$ is an unbounded solution to both (2) and (3) with an arbitrary function $\varphi: \mathbf{R} \rightarrow \mathbf{R}^{+}$subject to $\inf \{\varphi(t): t \in \mathbf{R}\} \geq 2|b||1-b|$. However, if we consider a fixed linear multiplicative functional $x^{*}: \mathcal{A} \rightarrow \mathrm{C}$ given by 


$$
x^{*}\left(\left[\begin{array}{ll}
a & 0 \\
0 & b
\end{array}\right]\right):=b
$$

then the superposition $\left(x^{*} \circ \bar{f}\right)(x)=b$ is constant and hence bounded, nevertheless

$$
\| \bar{f}(x+y)+\bar{f}(x-y)-2 \bar{f}(x) \bar{f}(y)||=2|b||1-b|
$$

fails to satisfy d'Alembert equation (1). This remark shows that Theorem 1.4 fails for the algebra $\mathcal{A}$ and $x^{*} \circ \bar{f}$.

Let $(G,+)$ be an abelian group and $\mathbf{C}$ the field of complex numbers throughout this article. Now, consider a function $f: G \rightarrow C$ satisfying approximately the functional equation

$$
f(x+y+z)+f(x+y-z)+f(y+z-x)+f(z+x-y)=4 f(x) f(y) f(z)
$$

for all $x, y, z \in G$. Given a mapping $f: G \rightarrow \mathbf{C}$, we are going to use a difference $D f(x$, $y, z): G^{3} \rightarrow \mathbf{C}$ as

$$
D f(x, y, z):=f(x+y+z)+f(x+y-z)+f(y+z-x)+f(z+x-y)=4 f(x) f(y) f(z)
$$

for all $x, y, z \in G$, which is called the remainder of Equation 4 and acts as a perturbation of Equation 4. The purpose of this article is to investigate the superstability of Equation 4 under the condition that the perturbing term $D f(x, y, z)$ is controlled by a function $\varphi(x), \varphi(y)$ or $\varphi(z)$. Moreover, we extend all superstability results for Equation 4 to the superstability on the commutative semisimple Banach algebra.

\section{Superstability of (4)}

In this section, we will investigate the superstability of the functional equation (4). The functional equation (4) is connected with the d'Alembert functional equation (1) as follows [19].

Lemma 2.1. A complex-valued function $f$ on an abelian group $G$ satisfies the functional equation

$$
f(x+y+z)+f(x+y-z)+f(y+z-x)+f(z+x-y)=4 f(x) f(y) f(z)
$$

for all $x, y, z \in G$ and $f(0) \geq 0$ if and only if $f$ satisfies the d'Alembert functional equation

$$
f(x+y)+f(x-y)=2 f(x) f(y)
$$

for all $x, y \in G$.

Theorem 2.2. Let $f: \in \mathbf{C}$ be a function and $\varphi: G \rightarrow \mathbf{R}^{+}:=[0, \infty)$ satisfy the inequality

$$
|D f(x, y, z)| \leq \phi(x)
$$

for all $x, y, z \in G$. Then, either $f$ is bounded or $f$ satisfies the functional equation

$$
f(x+y+z)+f(x+y-z)+f(y+z-x)+f(z+x-y)=4 f(x) f(y) f(z)
$$

for all $x, y, z \in G$.

Proof. If $f$ is unbounded, then we can choose a sequence $\left\{y_{n}\right\}_{n \in \mathbf{N}}$ in $G$ such that

$$
\left|f\left(y_{n}\right)\right|>1 \text { and } f\left(y_{n}\right) \mid \rightarrow \infty \quad \text { as } n \rightarrow \infty .
$$


Taking $y=z:=y_{n}$ in (5), we get

$$
\left|\left(f\left(2 y_{n}+x\right) f\left(2 y_{n}-x\right)\right)-f(x)\left(4 f\left(y_{n}\right)^{2}-2\right)\right| \leq \phi(x)
$$

for all $x, y, z \in G$. Dividing the above inequality by $\left|4 f\left(y_{n}\right)^{2}-2\right|$, then we get

$$
\left|f(x)-\frac{f\left(2 y_{n}+x\right)+f\left(2 y_{n}-x\right)}{4 f\left(y_{n}\right)^{2}-2}\right| \leq \frac{\phi(x)}{\left|4 f\left(y_{n}\right)^{2}-2\right|}
$$

for all $x, y, z \in G$. Passing to the limit as $n \rightarrow \infty$, we obtain the following:

$$
f(x)=\lim _{n \rightarrow \infty} \frac{f\left(2 y_{n}+x\right)+f\left(2 y_{n}-x\right)}{4 f\left(y_{n}\right)^{2}-2}
$$

for all $x \in G$. Note that the right-hand side of (7) is invariant under the inversion of $x$, we deduce that

$$
f(-x)=f(x) \quad \forall x \in G .
$$

Now, we will apply (7) to derive functional equation (6). Putting $(x, y, z):=\left(x, 2 y_{n}+\right.$ $y, z)$ in (5), we have

$$
\begin{array}{r}
\mid f\left(x+2 y_{n}+y+z\right)+f\left(x+2 y_{n}+y-z\right)+f\left(2 y_{n}+y+z-x\right)+f\left(z+x-2 y_{n}-y\right) \\
-4 f(x) f\left(2 y_{n}+y\right) f(z) \mid \leq \phi(x)
\end{array}
$$

for all $x, y, z \in G$. Letting $(x, y, z):=\left(x, 2 y_{n}-y, z\right)$ in (5), we have

$$
\begin{array}{r}
\mid f\left(x+2 y_{n}-y+z\right)+f\left(x+2 y_{n}-y-z\right)+f\left(2 y_{n}-y+z-x\right)+f\left(z+x-2 y_{n}+\gamma\right) \\
-4 f(x) f\left(2 y_{n}-y\right) f(z) \mid \leq \phi(x)
\end{array}
$$

for all $x, y, z \in G$. Combining (9) and (10) gives

$$
\begin{array}{r}
\mid\left(f\left(x+2 y_{n}+y+z\right)+f\left(z+x-2 y_{n}+y\right)\right)+\left(f\left(x+2 y_{n}+y-z\right)+f\left(2 y_{n}-y+z-x\right)\right) \\
+\left(f\left(2 y_{n}+y+z-x\right)+f\left(x+2 y_{n}-y-z\right)\right)+\left(f\left(z+x-2 y_{n}-y\right)+f\left(x+2 y_{n}-y+z\right)\right) \\
-4 f(x) f\left(2 y_{n}+y\right) f(z)+4 f(x) f\left(2 y_{n}-y\right) f(z) \mid \leq 2 \phi(x)
\end{array}
$$

for all $x, y, z \in G$. Using the fact (8) and applying the evenness of $f$, we see that

$$
\begin{aligned}
& \lim _{n \rightarrow \infty} \frac{f\left(x+2 y_{n}+y+z\right)+f\left(z+x-2 y_{n}+y\right)}{4 f\left(y_{n}\right)^{2}-2} \\
= & \lim _{n \rightarrow \infty} \frac{f\left(2 y_{n}+x+y+z\right)+f\left(2 y_{n}-(x+y+z)\right)}{4 f\left(y_{n}\right)^{2}-2} \\
= & f(x+y+z)
\end{aligned}
$$

for all $x, y, z \in G$. Similarly,

$$
\begin{aligned}
& \lim _{n \rightarrow \infty} \frac{f\left(x+2 y_{n}+y+z\right)+f\left(z+x-2 y_{n}+y\right)}{4 f\left(y_{n}\right)^{2}-2}=f(x+y-z), \\
& \lim _{n \rightarrow \infty} \frac{f\left(2 y_{n}+y+z-x\right)+f\left(x+2 y_{n}-y-z\right)}{4 f\left(y_{n}\right)^{2}-2}=f(y+z-x), \\
& \lim _{n \rightarrow \infty} \frac{f\left(z+x-2 y_{n}-y\right)+f\left(x+2 y_{n}-y+z\right)}{4 f\left(y_{n}\right)^{2}-2}=f(z+x-y)
\end{aligned}
$$

for all $x, y, z \in G$. Therefore, dividing inequality (11) by $\left|4 f\left(y_{n}\right)^{2}-2\right|$ and taking the limit as $n \rightarrow \infty$, we get 


$$
f(x+y+z)+f(x+y-z)+f(y+z-x)+f(z+x-y)=4 f(x) f(y) f(z)
$$

for all $x, y, z \in G$. This completes the proof.

Similarly we can prove that if the difference $D f(x, y, z)$ is bounded by $\varphi(y)$ or $\varphi(z)$, we obtain the same result as in Theorem 2.2.

Corollary 2.3. Let $\delta$ be a positive real number and let $f: G \rightarrow \mathbf{C}$ be a function satisfying the inequality

$$
|D f(x, y, z)| \leq \delta
$$

for all $x, y, z \in G$. Then, either $f$ is bounded or $f$ satisfies the functional equation

$$
f(x+y+z)+f(x+y-z)+f(y+z-x)+f(z+x-y)=4 f(x) f(y) f(z)
$$

for all $x, y, z \in G$.

\section{Extension to Banach algebra}

All the results in Section 2 can be extended to the superstability on the commutative semisimple Banach algebra. In this section, let $(G,+)$ be an abelian group, and $(E, \|$. I) be a commutative semisimple Banach algebra.

Theorem 3.1. Assume that $f: G \rightarrow E$ and $\varphi: G \rightarrow R^{+}$satisfy the inequality

$$
\|D f(x, y, z)\| \leq \phi(x)
$$

for all $x, y, z \in G$. For an arbitrary linear multiplicative functional $x^{*} \in E^{*}$, if the superposition $x^{*} \circ f$ is unbounded, then $f$ satisfies the functional equation

$$
f(x+y+z)+f(x+y-z)+f(y+z-x)+f(z+x-y)=4 f(x) f(y) f(z)
$$

for all $x, y, z \in G$.

Proof. Assume that (12) holds, and arbitrarily fix a linear multiplicative functional $x^{*}$ $\in E^{*}$. Let $\left\|x^{*}\right\|=1$ without loss of generality. Then, for every $x, y, z \in G$, we get

$$
\begin{aligned}
& \phi(x) \geq\|f(x+y+z)+f(x+y-z)+f(y+z-x)+f(z+x-y)-4 f(x) f(y) f(z)\| \\
&= \sup _{\left\|y^{*}\right\|=1}\left|y^{*}(f(x+y+z)+f(x+y-z)+f(y+z-x)+f(z+x-y)-4 f(x) f(y) f(x))\right| \\
& \geq \mid x^{*}(f(x+y+z))+x^{*}(f(x+y-z))+x^{*}(f(y+z-x))+x^{*}(f(z+x-y)) \\
&-4 x^{*}(f(x)) \cdot x^{*}(f(y)) \cdot x^{*}(f(z)) \mid,
\end{aligned}
$$

which states that the superposition $x^{*} \circ f: G \rightarrow \mathbf{C}$ yields a solution of the inequality (5) of Theorem 2.2. By assumption, since the superposition $x^{*} \circ f$ is unbounded, Theorem 2.2 shows that the superposition $x^{*} \circ f$ is a solution of Equation 6, namely,

$$
\begin{aligned}
& \left(x^{*} \circ f\right)(x+y+z)+\left(x^{*} \circ f\right)(x+y-z)+\left(x^{*} \circ f\right)(y+z-x)+\left(x^{*} \circ f\right)(z+x-y) \\
& =4\left(x^{*} \circ f\right)(x)\left(x^{*} \circ f\right)(y)\left(x^{*} \circ f\right)(z)
\end{aligned}
$$

for all $x, y, z \in G$. In other words, bearing the linear multiplicativity of $x^{*}$ in mind, for all $x, y, z \in G$, the difference $D f(x, y, z): G \times G \times G \rightarrow \mathbf{C}$ falls into the kernel of $x^{*}$. Therefore, in view of the unrestricted choice of $x^{*}$, we infer that

$$
D f(x, y, z) \in \bigcap\left\{\operatorname{ker} x^{*}: x^{*} \text { is a linear multiplicative member of } E^{*}\right\}
$$

for all $x, y, z \in G$. Since the algebra $E$ has been assumed to be semisimple, the last term of the above formula coincides with the singleton $\{0\}$, that is 


$$
D f(x, y, z)=0 \quad \forall x, y, z \in G
$$

as claimed. This completes the proof.

By the similar manner, we can prove that if the difference $D f(x, y, z)$ is bounded by $\varphi$ $(y)$ or $\varphi(z)$, we obtain the same result as in Theorem 3.1.

As results of superstability concerning Equation 4, we obtain application to harmonic functions satisfying the equation approximately.

Theorem 3.2. Let $f: \mathbf{R} \rightarrow \mathbf{C}$ and $\varphi: \mathbf{R} \rightarrow \mathbf{R}^{+}$satisfy the inequality

$$
\|D f(x, y, z)\| \leq \phi(x) \quad \forall x, y, z \in \mathbf{R} .
$$

If $f$ is an unbounded harmonic function, then there is a constant $\alpha \in \mathbf{C} \backslash \mathbf{R}$ such that $f(x)=\cos \alpha x$ and $f$ is a solution of the d'Alembert's functional equation (1).

Proof. By Theorem 2.2, $f$ satisfies the functional equation (4). Assume that $f$ is unbounded and $f(0)=0$. Putting $y=z:=0$ in (4), we get

$$
3 f(x)+f(-x)=4 f(x) f(0)^{2}=0
$$

for all $x \in \mathbf{R}$. Putting $x:=-x$ in (14) and then combining the equalities, we see that $f$ is odd and so $f(x)=0$ for all $x \in \mathbf{R}$. This is a contradiction. Therefore, $|f(0)|>0$. Hence, $f$ satisfies also the d'Alembert functional equation (1) by Lemma 2.1. It is well known that a harmonic solution $f: \mathbf{R} \rightarrow \mathbf{C}$ of the d'Alembert functional equation (1) has to have the form $f(x)=\cos \alpha x, \forall x \in \mathbf{R}$, where $\alpha$ is a complex number [18]. Since $f$ is unbounded, the constant $\alpha$ of that form falls into the set $\mathbf{C} \backslash \mathbf{R}$. This completes the proof.

Similarly, one can prove that if the difference $D f(x, y, z)$ is bounded by $\varphi(y)$ or $\varphi(z)$, one obtains the same result as in Theorem 3.2.

\section{Acknowledgements}

The authors would like to thank the referees for their valuable comments. The first author was partially supported by the Basic Research Program through the National Research Foundation of Korea funded by the Ministry of Education, Science and Technology (No. 2011-0002614). The second and third author of this study was partially supported by the Basic Science Research Program through the National Research Foundation of Korea (NRF) funded by the Ministry of Education, Science and Technology (Grant number: 2010-0010243).

\section{Author details}

'Department of Mathematics, Chungnam National University, 79 Daehangno, Yuseong-gu, Daejeon 305-764, Korea

${ }^{2}$ Department of Mathematics, Kangnam University, Yongin, Gyeonggi 446-702, Korea

\section{Authors' contributions}

All authors carried out the proof. All authors conceived of the study, and participated in its design and coordination.

All authors read and approved the final manuscript.

\section{Competing interests}

The authors declare that they have no competing interests.

Received: 18 May 2011 Accepted: 23 November 2011 Published: 23 November 2011

\section{References}

1. Ulam, SM: A Collection of Mathematical Problems. Interscience Publishers, New York (1960)

2. Hyers, DH: On the stability of the linear functional equations. Proc Nat Acad Sci USA. 27, 222-224 (1941). doi:10.1073/ pnas.27.4.222

3. Aoki, T: On the stability of the linear transformation in Banach spaces. J Math Soc Japan. 2, 64-66 (1950). doi:10.2969/ jmsj/00210064

4. Bourgin, DG: Classes of transformations and bordering transformations. Bull Am Math Soc. 57, 223-237 (1951). doi:10.1090/S0002-9904-1951-09511-7

5. Rassias, TM: On the stability of the linear mapping in Banach spaces. Proc Am Math Soc. 72, 297-300 (1978). doi:10.1090/S0002-9939-1978-0507327-1

6. Jung, SM: Hyers-Ulam-Rassias stability of functional equations. Dynam Syst Appl. 6, 541-566 (1997) 
7. Bodaghib, A, Alias, IA, Gordji, ME: On the stability of quadratic double centralizers and quadratic multipliers: a fixed point approach. J Inequal Appl 9 (2011). 2011, Article ID 957541

8. Gordji, ME, Khodae, H: On the generalized Hyers-Ulam-Rassias stability of quadratic functional equations. Abstr Appl Anal 11 (2009). 2009, Article ID 923476

9. Jung, SM: Hyers-Ulam stability of Fibonacci functional equation. Bull Iran Math Soc. 35, 217-227 (2009)

10. Khodaei, H, Rassias, TM: Approximately generalized additive functions in several variables. Int J Nonlinear Anal Appl. 1, 22-41 (2010)

11. Najati, A: On the stability of a quartic functional equation. J Math Anal Appl. 340, 569-574 (2008). doi:10.1016/j. jmaa.2007.08.048

12. Park, C: Generalized Hyers-Ulam stability of quadratic functional equations: a fixed point approach. Fixed Point Theory Appl 9 (2008). 2008, Article ID 493751

13. Pourpasha, MM, Rassias, JM, Saadati, R, Vaezpour, SM: A fixed point approach to the stability of Pexider quadratic functional equation with involution. J Inequal Appl 18 (2010). 2010, Article ID 839639

14. Rahimi, A, Najati, A, Bae, JH: On the asymptoticity aspect of Hyers-Ulam stability of quadratic mappings. J Inequal Appl 14 (2010). 2010, Article ID 454875

15. Saadati, R, Zohdi, MM, Vaezpour, SM: Nonlinear L-random stability of an ACQ-functional equation. J Inequal Appl 23 (2011). 2011, Article ID 194394

16. Baker, JA, Lawrence, J, Zorzitto, F: The stability of the equation $f(x+y)=f(x) f(y)$. Proc Am Math Soc. 74, 242-246 (1979)

17. Baker, JA: The stability of the cosine equation. Proc Nat Acad Sci USA. 3, 411-416 (1980)

18. Badora, R, Ger, R: On some trigonometric functional inequalities. In: Daroczy, Z, Pales Z (eds.) Functional EquationsResults and Advances. pp. 3-15. Kluwer Academic Publishers, Dordrecht (2002)

19. Nakmahschalasint, P: The stability of a cosine functional equation. KMITL Sci J. 1, 49-53 (2007)

doi:10.1186/1029-242X-2011-118

Cite this article as: Kim et al: Superstability of approximate d'Alembert harmonic functions. Journal of Inequalities and Applications 2011 2011:118.

\section{Submit your manuscript to a SpringerOpen ${ }^{\odot}$} journal and benefit from:

- Convenient online submission

- Rigorous peer review

- Immediate publication on acceptance

- Open access: articles freely available online

- High visibility within the field

- Retaining the copyright to your article

Submit your next manuscript at $\gg$ springeropen.com 